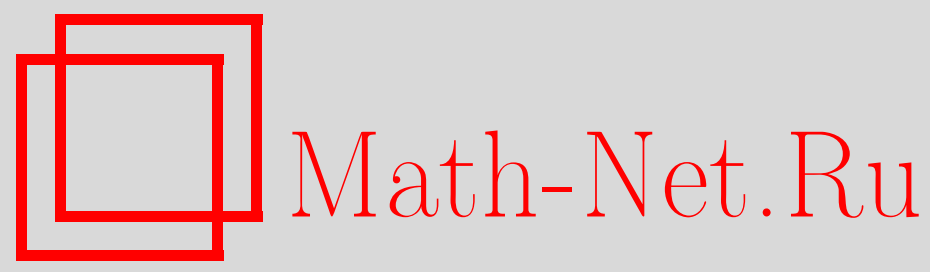

В. С. Матвеев, Собственные значения отображения Синюкова для геодезически эквивалентных метрик глобально упорядочены, Матем. заметки, 2005, том 77, выпуск $3,412-423$

DOI: https://doi.org/10.4213/mzm2502

Использование Общероссийского математического портала Math-Net.Ru подразумевает, что вы прочитали и согласны с пользовательским соглашением http://www . mathnet.ru/rus/agreement

Параметры загрузки:

IP : 3.91 .87 .62

26 апреля 2023 г., 12:50:04

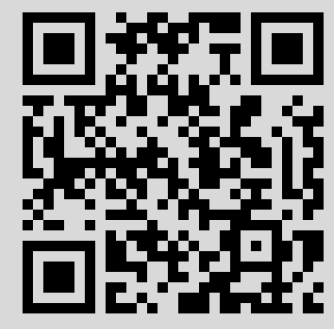




\section{СОБСТВЕННЫЕ ЗНАЧЕНИЯ ОТОБРАЖЕНИЯ СИНЮКОВА ДЛЯ ГЕОДЕЗИЧЕСКИ ЭКВИВАЛЕНТНЫХ МЕТРИК ГЛОБАЛЬНО УПОРЯДОЧЕНЫ}

B. С. Матвеев

Пусть все геодезические двух римановых метрик $g$ и $\bar{g}$, заданных на одном (связном, геодезически полном) многообразии $M^{n}$, совпадают. В каждой точке $x \in M^{n}$ рассмотрим совместные собственные значения $\rho_{1}, \rho_{2}, \ldots, \rho_{n}$ этих метрик (предполагая $\left.\rho_{1} \geqslant \rho_{2} \geqslant \cdots \geqslant \rho_{n}\right)$ и числа

$$
\lambda_{i}=\left(\rho_{1} \rho_{2} \cdots \rho_{n}\right)^{1 /(n+1)} \frac{1}{\rho_{i}} .
$$

Мы покажем, что числа $\lambda_{i}$ упорядочены на всем многообразии: для любых двух точек $x$ и $y$ многообразия, число $\lambda_{k}(x)$ всегда не больше числа $\lambda_{k+1}(y)$. Если $\lambda_{k}(x)=$ $\lambda_{k+1}(y)$, то существует точка $z \in M^{n}$, в которой $\lambda_{k}(z)=\lambda_{k+1}(z)$. Если многообразие замкнуто и если все совместные собственные значения этих метрик различны в каждой точке, то многообразие накрывается тором.

Библиография: 14 названий.

1. Формулировки. Пусть $g, \bar{g}$ - две $C^{2}$-гладкие римановы метрики на $n$-мерном многообразии $M^{n}$.

ОПРЕДЕЛЕНИЕ 1. Метрики $g$ и $\bar{g}$ называются геодезически әквивалентными, если все их геодезические, рассматриваемые как непараметризованные кривые, совпадают.

Рассмотрим послойно-линейное отображение

$$
G: T M^{n} \rightarrow T M^{n}
$$

заданное тензором

$$
G_{j}^{i}=g^{i \alpha} \bar{g}_{\alpha j}
$$

В инвариантных терминах для любой точки $x \in M^{n}$ ограничение послойно-линейного отображения $G$ на касательное пространство $T_{x} M^{n}$ является таким линейным преобразованием пространства $T_{x} M^{n}$, что для любых двух векторов $u, v \in T_{x} M^{n}$ справедливо равенство

$$
g(G(u), v)=\bar{g}(u, v)
$$

где $g(\cdot, \cdot)$ и $\bar{g}(\cdot, \cdot)$ обозначают скалярные произведения в метриках $g$ и $\bar{g}$ соответственHO.

Работа выполнена при финансовой поддержке European Post-Doctoral Institute for Mathematical Sciences. 
ОПРЕДЕЛЕНИЕ 2. Отображением Синюкова для пары метрик $g, \bar{g}$ называется послойно-линейное отображение

$$
B: T M^{n} \rightarrow T M^{n}
$$

заданное тензором

$$
B_{j}^{i}=\left(\frac{\operatorname{det}(\bar{g})}{\operatorname{det}(g)}\right)^{1 /(n+1)} \bar{g}^{i \alpha} g_{\alpha j}
$$

В инвариантных терминах

$$
B=(\operatorname{det}(G))^{1 /(n+1)} G^{-1}
$$

Отображение Синюкова и его характеристические инварианты играют важную роль в теории геодезически эквивалентных метрик (см., например, обзорную статью [1]). В частности, если метрики $g, \bar{g}$ геодезически эквивалентны, то метрики $g_{B}=g_{i \alpha} B_{j}^{\alpha}$, $\bar{g}_{B}=\bar{g}_{i \alpha} B_{j}^{\alpha}$ тоже геодезически эквивалентны (см. [2]).

Собственными значениями отображсения $G$ в точке $x \in M^{n}$ будем назьвать собственные значения ограничения послойно-линейного отображения $G$ на касательное пространство $T_{x} M^{n}$, т.е. корни многочлена

$$
\left.\operatorname{det}(G-\rho E)\right|_{x}=\left.\left(\frac{1}{\operatorname{det}(g)} \operatorname{det}(\bar{g}-\rho g)\right)\right|_{x}
$$

Собственные значения отображения $G$ называются также совместными собственными значениями метрик $g, \bar{g}$.

Аналогично, собственными значениями послойно-линейного отображсния $B$ в точке $x \in M^{n}$ мы будем называть корни многочлена

$$
\left.\operatorname{det}(B-\lambda E)\right|_{x}=\left.\left(\left(\frac{1}{\operatorname{det}(\bar{g})}\right)^{1 /(n+1)} \operatorname{det}\left(\frac{g}{(\operatorname{det}(g))^{1 /(n+1)}}-\lambda \frac{\bar{g}}{(\operatorname{det}(\bar{g}))^{1 /(n+1)}}\right)\right)\right|_{x}
$$

Так как метрики $g, \bar{g}$ положительно определены, в каждой точке $x \in M^{n}$ все собственные значения послойно-линейных отображений $G$ и $B$ вещественны и положительны. $\mathrm{C}$ учетом кратности их количество равно $n$. Пусть $\rho_{1}(x), \rho_{2}(x), \ldots, \rho_{n}(x)$ - упорядоченный по убыванию набор собственных значений послойно-линейного отображения $G$ в точке $x \in M^{n}$ :

$$
\rho_{1}(x) \geqslant \rho_{2}(x) \geqslant \cdots \geqslant \rho_{n}(x) .
$$

Пусть $\lambda_{1}(x), \lambda_{2}(x), \ldots, \lambda_{n}(x)$ - упорядоченный по возрастанию набор собственных значений послойно-линейного отображения $B$ в точке $x \in M^{n}$ :

$$
\lambda_{1}(x) \leqslant \lambda_{2}(x) \leqslant \cdots \leqslant \lambda_{n}(x) .
$$

Числа $\lambda_{i}(x)$ и $\rho_{i}(x)$ очевидно связаны соотношениями

$$
\lambda_{i}(x)=\left(\rho_{1}(x) \rho_{2}(x) \cdots \rho_{n}(x)\right)^{1 /(n+1)} \frac{1}{\rho_{i}(x)}, \quad \rho_{i}(x)=\frac{1}{\lambda_{1}(x) \lambda_{2}(x) \cdots \lambda_{n}(x)} \frac{1}{\lambda_{i}(x)} .
$$


ТЕОРемА 1. Пусть метрики $g, \bar{g}$ геодезически әквивалентны на многообразии $M^{n}$. Пусть многообразие $M^{n}$ связно и геодезически полно по отношению $к$ метрике g. Тогда для любых точек $x, y \in M^{n}$, для любого числа $i \in\{1,2, \ldots, n-1\}$

$$
\lambda_{i}(x) \leqslant \lambda_{i+1}(y) .
$$

При этом выполнение неравенства $\lambda_{i}(x)<\lambda_{i+1}(x)$ для какой-нибудь точки $x \in M^{n}$ влечет справедливость неравенства $\lambda_{i}(z)<\lambda_{i+1}(z)$ для почти всех точек $z$ многообразия $M^{n}$.

Другими словами, собственные значения отображения Синюкова для геодезически эквивалентных метрик глобально упорядочены на всем связном геодезически полном (по отношению к любой из метрик) многообразии.

СЛЕдСТВИЕ 1 [3]. Пусть метрики $g, \bar{g}$ геодезически әквивалентны на многообразии $M^{n}$. Пусть многообразие $M^{n}$ связно и геодезически полно по отношению $\kappa$ метрике $g$. Тогда если метрики $g$ и $\bar{g}$ пропорииональны в каждой точке некоторого подмножества $U \subset M^{n}$ полохительной меры, то $\bar{g}=$ Const $\cdot g$.

ТЕОРема 2. Пусть метрики $g, \bar{g}$ геодезически әквивалентны на многообразии $M^{n}$. Пусть многообразие $M^{n}$ связно и геодезически полно по отношению $\kappa$ метрике $g$. Тогда если для точек $x, y \in M^{n}$, для вещественного числа $\lambda$ и для челого числа $i \in\{1,2, \ldots, n-1\}$ выполняется равенство

$$
\lambda_{i}(x)=\lambda_{i+1}(y)=\lambda,
$$

то существует такая точка $z \in M^{n}$, что $\lambda_{i}(z)=\lambda_{i+1}(z)=\lambda$.

ТЕОремА 3. Пусть метрики $g, \bar{g}$ геодезически әквивалентны на связном замкнутом многообразии $M^{n}$. Пусть все собственные значения послойно-линейного отображсения $G$ различны в каждой точке многообразия. Тогда многообразие $M^{n}$ накрывается тором $T^{n}$.

ЗАмЕчАниЕ 1 . В двумерном случае теорема 3 доказана в [4], а теоремы 1,2 вытекают из классификации квадратично интегрируемьх геодезических потоков на полных поверхностях, полученной в [5], [6]. В трехмерном случае теоремы 1-3 доказаны в [7]-[9].

Основным инструментом доказательств теорем 1-3 является следующая теорема, доказанная в [10], [11] (см. также [12], [13]).

Для каждой точки $x \in M^{n}$ рассмотрим характеристический многочлен

$$
\left.\operatorname{det}(G-\rho E)\right|_{x}=c_{0} \rho^{n}+c_{1} \rho^{n-1}+\cdots+c_{n} .
$$

Коэффициенты этого многочлена являются $C^{2}$-гладкими функциями на многообразии, причем $c_{0} \equiv(-1)^{n}$.

Рассмотрим послойно-линейные отображения

$$
S_{0}, S_{1}, \ldots, S_{n-1}: T M^{n} \rightarrow T M^{n}
$$

заданные общей формулой

$$
S_{k}=\left(\frac{\operatorname{det}(g)}{\operatorname{det}(\bar{g})}\right)^{(k+2) /(n+1)} \sum_{i=0}^{k} c_{i} G^{k-i+1} .
$$


Компоненты тензора, соответствуюшего послойно-линейному отображению $S_{k}$, будем обозначать $\left(S_{k}\right)_{j}^{i}$.

Рассмотрим функции $I_{k}: T^{*} M^{n} \rightarrow \mathbb{R}, k=0,1, \ldots, n-1$, заданные общей формулой

$$
I_{k}(x, p)=g^{\alpha j}\left(S_{k}\right)_{\alpha}^{i} p_{i} p_{j}
$$

В инвариантных терминах функции $I_{k}$ задаются следующим образом. С помощюю метрики $g$ отождествим канонически касательное и кокасательное расслоения многообразия $M^{n}$. Тогда для любой точки $x \in M^{n}$, для любого ковектора $\xi \in T_{x}^{*} M^{n} \cong T_{x} M^{n}$ значение функции $I_{k}$ на нем задается

$$
I_{k}(x, \xi)=g\left(S_{k}(\xi), \xi\right)
$$

Рассмотрим каноническую симплектическую форму $\omega=d p_{i} \wedge d x^{i}$ на кокасательном расслоении $T^{*} M^{n}$. Под геодезическим потоком метрики $g$ мы будем понимать гамильтонову систему на кокасательном расслоении $T^{*} M^{n}$, задаваемую гамильтонианом $H(x, \xi)=\frac{1}{2} g(\xi, \xi)$. Известно, что траектории геодезического потока метрики $g$ проектируются в геодезические метрики $g$. А именно, пусть кривая $\gamma: \mathbb{R} \rightarrow M^{n}$ является геодезической метрики $g$. Тогда кривая $(\gamma, \dot{\gamma}): \mathbb{R} \rightarrow T M^{n} \cong T^{*} M^{n}$, где $\dot{\gamma}(t) \in T_{\gamma(t)} M^{n} \cong$ $T_{\gamma(t)}^{*} M^{n}$ суть вектор скорости кривой $\gamma$ в точке $\gamma(t)$, является траекторией геодезического потока метрики $g$.

ЗАмечАниЕ 2 . По теореме Гамильтона-Кэли отображение $-S_{n-1}$ является тождественным. Следовательно, функция $-\frac{1}{2} I_{n-1}$ совпадает с гамильтонианом геодезического потока метрики $g$.

ТЕОРемА 4. Если метрики $g$ и $\bar{g}$ на многообразии $M^{n}$ геодезически эквивалентны, то функиии $I_{k}$ коммутируют.

Другими словами, если метрики $g$ и $\bar{g}$ геодезически эквивалентны, то функции $I_{k}$ являются коммутирующими интегралами геодезического потока метрики $g$.

ЗАмЕчАниЕ 3. Квантовая версия теоремы 4 доказана в [3].

Для доказательства теоремы 1 мы рассмотрим следующую функцию на прямом произведении $\mathbb{R} \times T^{*} M^{n}$ :

$$
F_{t}=t^{n-1} I_{n-1}+t^{n-2} I_{n-2}+\cdots+I_{0}
$$

Для каждой точки $(x, \xi) \in T^{*} M^{n}$ функция $(2)$ является многочленом по $t$. Его корни будем обозначать $t_{1}(x, \xi), t_{2}(x, \xi), \ldots, t_{n-1}(x, \xi)$, предполагая

$$
t_{1}(x, \xi) \leqslant t_{2}(x, \xi) \leqslant \cdots \leqslant t_{n-1}(x, \xi) .
$$

Мы покажем, что для каждой точки $(x, \xi) \in T^{*} M^{n}$ вьполняются неравенства

$$
\lambda_{i}(x) \leqslant t_{i}(x, \xi) \leqslant \lambda_{i+1}(x) .
$$

Так как коэффициенты многочлена (2) сохраняются вдоль геодезической, его корни тоже сохраняются.

Соединяя произвольные точки $x, y \in M^{n}$ геодезической, получаем

$$
\lambda_{i}(x) \leqslant t_{i} \leqslant \lambda_{i+1}(y)
$$


откуда вытекает теорема 1.

Для доказательства теоремы 2 мы покажем, что геодезическая $\gamma$, соединяющая точки $x$ и $y$, для которых

$$
\lambda_{i}(x)=\lambda_{i+1}(y)=\lambda,
$$

проходит через некоторую точку $z \in M^{n}$, в которой $\lambda_{i}(z)=\lambda_{i+1}(z)=\lambda$. Фактически мы покажем, что в каждой точке $w$ этой геодезической либо $\lambda_{i}(w)=\lambda$, либо $\lambda_{i+1}(w)=\lambda$, либо реализуются обе эти возможности. Так как множества

$$
\begin{aligned}
& \Gamma_{0}=\left\{w \in \gamma: \lambda_{i}(w)=\lambda\right\}, \\
& \Gamma_{1}=\left\{w \in \gamma: \lambda_{i+1}(w)=\lambda\right\},
\end{aligned}
$$

очевидно. замкнуты и их объединение совпадает с геодезической $\gamma$, то их пересечение, т.е. множество точек $z \in \gamma$, в которых $\lambda_{i}(z)=\lambda_{i+1}(z)=\lambda$, непусто.

Если совместные собственные значения метрик $g, \bar{g}$ различны в каждой точке многообразия, то интегралы $I_{k}$ из теоремы 4 функционально независимы. Если к тому же многообразие замкнуто, то типическая связная компонента поверхности уровня $\left\{I_{0}=\right.$ const $_{0}, I_{1}=$ const $_{1}, \ldots, I_{n-1}=$ const $\left._{n-1}\right\}$ является $n$-мерным тором $T^{n}$, которьй назьвается тором Лиувилля. Мы покажем, что в предположениях теоремы для подходящих констант const $_{0}$, const $_{1}, \ldots$, const $_{n-1}$ ограничение проекции $\pi: T^{*} M^{n} \rightarrow M^{n}$, $\pi(x, \xi)=x$, на соответствуюший тор Лиувилля не имеет особых точек и, тем самым, является накрытием многообразия $M^{n}$.

Автор благодарен П. Топалову и Ур. Хаменштадт за ценные обсуждения, А. В. Аминовой, А. В. Болсинову, А. С. Мищенко и А. Т. Фоменко за внимание к работе.

2. Доказательства. Рассмотрим функцию

$$
\begin{gathered}
F_{t}: \mathbb{R} \times T^{*} M^{n} \rightarrow \mathbb{R} \\
F_{t}(x, \xi)=t^{n-1} I_{n-1}(x, \xi)+t^{n-2} I_{n-2}(x, \xi)+\cdots+I_{0}(x, \xi) .
\end{gathered}
$$

Для каждой точки $(x, \xi) \in T^{*} M^{n} F_{t}$ является многочленом по $t$ с неположительньм старшим коэффициентом. Если функции $I_{0}, I_{1}, \ldots, I_{n-1}$ являются интегралами геодезического потока метрики $g$, то для каждого фиксированного $t$ функция $F_{t}$ является интегралом геодезического потока метрики $g$.

Лемма 1. Верны следующие утверждения:

1) для любой точки $(x, \xi) \in T^{*} M^{n}$ все корни многочлена $F_{t}$ вещественны $и$ положительны;

2) для любой точки $(x, \xi) \in T^{*} M^{n}$, для любого $i \in\{1,2, \ldots, n-1\}$

$$
\lambda_{i}(x) \leqslant t_{i}(x, \xi) \leqslant \lambda_{i+1}(x) .
$$

ДокАЗАТЕЛЬСтво. Фиксируем точку $x \in M^{n}$. Для простоты будем писать $\lambda_{i}, \rho_{i}$, $t_{i}(\xi)$ вместо $\lambda_{i}(x), \rho_{i}(x), t_{i}(x, \xi)$. Подходящей заменой координат в кокасательном пространстве $T_{x}^{*} M^{n}$ первая метрика приводится к единично-диагональному виду, а вторая - к диагональному

$$
\operatorname{diag}\left(\rho_{1}, \rho_{2}, \ldots, \rho_{n}\right) .
$$


Обозначим через $\sigma_{j}$ элементарньй симметрический многочлен степени $j$ от $n$ переменных $\lambda_{1}, \lambda_{2}, \ldots, \lambda_{n}$. Обозначим через $\sigma_{j}\left(\check{\lambda}_{i}\right)$ элементарный симметрический многочлен степени $j$ от $n-1$ переменной $\lambda_{1}, \lambda_{2}, \ldots, \lambda_{i-1}, \lambda_{i+1}, \ldots, \lambda_{n}$. Покажем по индукции, что матрица отображения $S_{k}$ задается

$$
S_{k}=(-1)^{n-k} \operatorname{diag}\left(\sigma_{n-k-1}\left(\check{\lambda}_{1}\right), \sigma_{n-k-1}\left(\check{\lambda}_{2}\right), \ldots, \sigma_{n-k-1}\left(\check{\lambda}_{n}\right)\right) .
$$

Очевидно, коэффициенты $c_{k}$ характеристического полинома (1) задаются формулой

$$
c_{k}=(-1)^{n-k} \frac{\sigma_{n-k}}{\left(\lambda_{1} \lambda_{2} \cdots \lambda_{n}\right)^{k+1}}
$$

В частности,

$$
\operatorname{det}(G)=c_{n}=\frac{1}{\left(\lambda_{1} \lambda_{2} \cdots \lambda_{n}\right)^{n+1}} .
$$

База индукции: для $k=0$ имеем

$$
\begin{aligned}
S_{0} & =\left(\frac{1}{\operatorname{det}(G)}\right)^{2 /(n+1)} c_{0} G \\
& =(-1)^{n}\left(\lambda_{1} \lambda_{2} \cdots \lambda_{n}\right)^{2} \operatorname{diag}\left(\frac{1}{\lambda_{1}\left(\lambda_{1} \lambda_{2} \cdots \lambda_{n}\right)}, \frac{1}{\lambda_{2}\left(\lambda_{1} \lambda_{2} \cdots \lambda_{n}\right)}, \ldots, \frac{1}{\lambda_{n}\left(\lambda_{1} \lambda_{2} \cdots \lambda_{n}\right)}\right) \\
& =(-1)^{n} \operatorname{diag}\left(\lambda_{2} \lambda_{3} \cdots \lambda_{n}, \lambda_{1} \lambda_{3} \cdots \lambda_{n}, \ldots, \lambda_{1} \lambda_{2} \cdots \lambda_{n-2} \lambda_{n}, \lambda_{1} \lambda_{2} \cdots \lambda_{n-2} \lambda_{n-1}\right) \\
& =(-1)^{n} \operatorname{diag}\left(\sigma_{n-1}\left(\check{\lambda}_{1}\right), \sigma_{n-1}\left(\check{\lambda}_{2}\right), \ldots, \sigma_{n-1}\left(\check{\lambda}_{n}\right)\right) .
\end{aligned}
$$

Индукционньй переход: пусть равенство (5) вьполняется для $S_{k-1}$. Тогда для $S_{k}$ имеем

$$
\begin{aligned}
& S_{k}=\left(\frac{1}{\operatorname{det}(G)}\right)^{1 /(n+1)} G\left(S_{k-1}+\left(\frac{1}{\operatorname{det}(G)}\right)^{(k+1) /(n+1)} c_{k} E\right) \\
&= \operatorname{diag}\left(\frac{1}{\lambda_{1}}, \frac{1}{\lambda_{2}}, \ldots, \frac{1}{\lambda_{n}}\right) \\
& \times \operatorname{diag}\left((-1)^{n-k}\left(\sigma_{n-k}-\sigma_{n-k}\left(\check{\lambda}_{1}\right)\right),(-1)^{n-k}\left(\sigma_{n-k}-\sigma_{n-k}\left(\check{\lambda}_{2}\right)\right), \ldots,\right. \\
&\left.(-1)^{n-k}\left(\sigma_{n-k}-\sigma_{n-k}\left(\check{\lambda}_{n}\right)\right)\right) .
\end{aligned}
$$

Используя $\left(\sigma_{l}-\sigma_{l}\left(\check{\lambda}_{i}\right)\right)=\lambda_{i} \sigma_{l-1}\left(\check{\lambda}_{i}\right)$, получаем, что матрища $S_{k}$ является диагональной матрицей

$$
(-1)^{n-k} \operatorname{diag}\left(\sigma_{n-k-1}\left(\check{\lambda}_{1}\right), \sigma_{n-k-1}\left(\check{\lambda}_{2}\right), \ldots, \sigma_{n-k-1}\left(\check{\lambda}_{n}\right)\right) .
$$

Равенство (5) доказано.

Таким образом, имеем

$$
I_{k}=(-1)^{n-k} \sum_{i=1}^{n} \sigma_{n-k-1}\left(\check{\lambda}_{i}\right) p_{i}^{2}
$$

Используя следующее очевидное равенство:

$$
\sum_{k=0}^{n-1} t^{k} \sigma_{n-k-1}\left(\check{\lambda}_{i}\right)(-1)^{n-k}=-\left(t-\lambda_{1}\right)\left(t-\lambda_{2}\right) \cdots\left(t-\lambda_{i-1}\right)\left(t-\lambda_{i+1}\right) \cdots\left(t-\lambda_{n}\right),
$$


получаем, что многочлен $F_{t}$ задается формулой

$$
F_{t}=\sum_{k=0}^{n-1} t^{k} I_{k}=\sum_{k=0}^{n-1}\left(\sum_{i=1}^{n} t^{k}(-1)^{n-k} \sigma_{n-k-1}\left(\check{\lambda}_{i}\right) p_{i}^{2}\right)=-\sum_{i=1}^{n} \Pi_{i}(t) p_{i}^{2},
$$

где функции $\Pi_{i}(t)$ задаются

$$
\Pi_{i}(t)=\left(t-\lambda_{1}\right)\left(t-\lambda_{2}\right) \cdots\left(t-\lambda_{i-1}\right)\left(t-\lambda_{i+1}\right) \cdots\left(t-\lambda_{n}\right) .
$$

Мы видим, что $F_{t}$ является многочленом степени $n-1$ по $t$ с коэффищиентами, зависящими от ковектора $\xi=\left(p_{1}, p_{2}, \ldots, p_{n}\right)$. Таким образом, нам необходимо показать, что все корни $t_{i}(\xi)$ многочлена (6) вещественны и что для любого ковектора $\xi$ корень $t_{i}(\xi)$ лежит на отрезке $\left[\lambda_{i}, \lambda_{i+1}\right]$. Из соображений непрерывности достаточно показать это в предположении, что все $p_{i}$ отличны от нуля и все собственные значения $\lambda_{i}$ различны. Тогда для любого $i \in\{1,2, \ldots, n-1\}$ знаки значений многочлена $F_{t}$ при $t=\lambda_{i}$ и при $t=\lambda_{i+1}$ различны. Действительно, при $i \neq j$ имеем

$$
\Pi_{i}\left(\lambda_{j}\right)=0 .
$$

Следовательно, значение $(-1)^{n-i+1} F_{\lambda_{i}}$ задается формулой

$$
\begin{aligned}
(-1)^{n-i-1} F_{\lambda_{i}} & =(-1)^{n-i}\left(\lambda_{i}-\lambda_{1}\right)\left(\lambda_{i}-\lambda_{2}\right) \cdots\left(\lambda_{i}-\lambda_{i-1}\right)\left(\lambda_{i}-\lambda_{i+1}\right) \cdots\left(\lambda_{i}-\lambda_{n}\right) p_{i}^{2} \\
& =\left(\lambda_{i}-\lambda_{1}\right)\left(\lambda_{i}-\lambda_{2}\right) \cdots\left(\lambda_{i}-\lambda_{i-1}\right)\left(\lambda_{i+1}-\lambda_{i}\right) \cdots\left(\lambda_{n}-\lambda_{i}\right) p_{i}^{2} .
\end{aligned}
$$

Так как $\lambda_{i}>\lambda_{j}$ для $i>j$ и так как, по предположению, $p_{i}^{2}>0$, то число (7) положительно и, следовательно, знаки значений многочлена $F_{t}$ при $t=\lambda_{i}$ и при $t=\lambda_{i+1}$ различны. Следовательно, существует хотя бы один корень $t$ многочлена $F_{t}$ внутри открытого интервала ] $\lambda_{i}, \lambda_{i+1}$ [. Степень многочлена $F_{t}$ равна $n-1$, всего мы имеем $n-1$ непересекаюшихся интервалов, на каждом из которых лежит корень.

Лемма доказана.

Лемма 2. Пусть точка $x \in M^{n}$. Рассмотрим произвольное число $\tau>0$. Если для любого әлемента $\xi$ некоторого подмножества $U \subset T_{x}^{*} M^{n}$ положительной меры число $\tau$ является корнем кратности $k \geqslant 1$ многочлена $F_{t}(x, \xi)$, то число $\tau$ является собственным значением кратности $k+1$ отображсения $B$ в точке $x . B$ частности, если для любого әлемента $\xi \in U^{n}$ справедливо равенство $t_{i}(x, \xi)=\tau$, $m o \tau=\lambda_{i}(x)=\lambda_{i+1}(x)$.

ДокАЗАТЕЛЬСТВО. Пусть для любого ковектора $\xi=\left(p_{1}, p_{2}, \ldots, p_{n}\right) \in U \subset T_{x}^{*} M^{n}$ число $\tau$ является корнем многочлена $F_{t}(x, \xi)$. Обозначим через

$$
\lambda_{1}<\lambda_{2}<\cdots<\lambda_{m}
$$

различные собственные значения отображения $B$ в точке $x$. Кратность собственного значения $\lambda_{i}$ обозначим через $k_{i}$, так что $k_{1}+k_{2}+\cdots+k_{m}=n$. В этих обозначениях функции $\Pi_{i}(t)$ из формулы (6) переписьваются как

$$
\Pi_{i}(t)=\left(t-\lambda_{1}\right)^{k_{1}}\left(t-\lambda_{2}\right)^{k_{2}} \cdots\left(t-\lambda_{i}\right)^{k_{i}-1} \cdots\left(t-\lambda_{m}\right)^{k_{m}},
$$


и, следовательно, многочлен $F_{t}$ задается формулой

$$
F_{t}=-\sum_{i=1}^{m} \Pi_{i}(t) p_{i}^{2}=-\left(t-\lambda_{1}\right)^{k_{1}-1}\left(t-\lambda_{2}\right)^{k_{2}-1} \cdots\left(t-\lambda_{m}\right)^{k_{m}-1}\left(\sum_{i=1}^{m} \widetilde{\Pi}_{i}(t) P_{i}^{2}\right)
$$

где $\widetilde{\Pi}_{i}: \mathbb{R} \rightarrow \mathbb{R}, P_{i}^{2}: T_{x}^{*} M^{n} \rightarrow \mathbb{R}, i \in\{1,2, \ldots, m\}$, суть следующие функции:

$$
\begin{gathered}
\widetilde{\Pi}_{i}(t)=\left(t-\lambda_{1}\right)\left(t-\lambda_{2}\right) \cdots\left(t-\lambda_{i-1}\right)\left(t-\lambda_{i+1}\right) \cdots\left(t-\lambda_{m}\right) \\
P_{1}^{2}=p_{1}^{2}+p_{2}^{2}+\cdots+p_{k_{1}}^{2}, \quad P_{2}^{2}=p_{k_{1}+1}^{2}+\cdots+p_{k_{1}+k_{2}}^{2}, \quad \cdots \\
P_{m}^{2}=p_{k_{1}+k_{2}+\cdots+k_{m-1}+1}^{2}+\cdots+p_{n}^{2}
\end{gathered}
$$

Для каждого $\tau \in \mathbb{R}$, по крайней мере, одно из чисел $\Pi_{i}(\tau)$ отлично от нуля, так что множество

$$
Q_{\tau}:=\left\{\left(p_{1}, p_{2}, \ldots, p_{n}\right) \in T_{x}^{*} M^{n}: \sum_{i=1}^{m} \widetilde{\Pi}_{i}(\tau) P_{i}^{2}=0\right\}
$$

является квадрикой и, следовательно, имеет меру нуль.

Таким образом, если подмножество $U$ имеет положительную меру, то $U \backslash Q_{\tau}$ непусто. Следовательно, число $\tau$ является корнем многочлена

$$
\left(t-\lambda_{1}\right)^{k_{1}-1}\left(t-\lambda_{2}\right)^{k_{2}-1} \cdots\left(t-\lambda_{m}\right)^{k_{m}-1}
$$

кратности $k$. Лемма доказана.

ДокАЗАТЕЛЬСТво ТЕОРЕМЫ 1 . Пусть метрики $g, \bar{g}$ геодезически эквивалентны на многообразии $M^{n}$. Рассмотрим произвольную геодезическую $\gamma(\tau)$ (для первой метрики). Рассмотрим соответствующую траекторию геодезического потока метрики $g$. Канонически отождествим (с помощю метрики $g$ ) касательное и кокасательное расслоения к многообразию $M^{n}$. Тогда траектория геодезического потока, соответствующая геодезической $\gamma$, является кривой

$$
(\gamma, \dot{\gamma}): \mathbb{R} \rightarrow T M^{n} \cong T^{*} M^{n}, \quad(\gamma, \dot{\gamma})(\tau)=(\gamma(\tau), \dot{\gamma}(\tau)),
$$

где $\dot{\gamma}(\tau) \in T_{\gamma(\tau)} M^{n}$ суть вектор скорости геодезической в точке $\gamma(\tau) \in M^{n}$. Рассмотрим две произвольные точки $\gamma\left(\tau_{1}\right)$ и $\gamma\left(\tau_{2}\right)$, лежащие на геодезической $\gamma$. По лемме 1 для любого $i \in\{1,2, \ldots, n-1\}$ имеем

$$
\begin{aligned}
& \lambda_{i}\left(\gamma\left(\tau_{1}\right)\right) \leqslant t_{i}\left(\gamma\left(\tau_{1}\right), \dot{\gamma}\left(\tau_{1}\right)\right) \leqslant \lambda_{i+1}\left(\gamma\left(\tau_{1}\right)\right), \\
& \lambda_{i}\left(\gamma\left(\tau_{2}\right)\right) \leqslant t_{i}\left(\gamma\left(\tau_{2}\right), \dot{\gamma}\left(\tau_{2}\right)\right) \leqslant \lambda_{i+1}\left(\gamma\left(\tau_{2}\right)\right) .
\end{aligned}
$$

Так как коэффициенты $I_{0}, I_{1}, \ldots, I_{n-1}$ многочлена $F_{t}$ постоянны на траекториях геодезического потока, то $t_{i}\left(\gamma\left(\tau_{1}\right), \dot{\gamma}\left(\tau_{1}\right)\right)=t_{i}\left(\gamma\left(\tau_{2}\right), \dot{\gamma}\left(\tau_{2}\right)\right)$. Следовательно,

$$
\lambda_{i}\left(\gamma\left(\tau_{1}\right)\right) \leqslant \lambda_{i+1}\left(\gamma\left(\tau_{2}\right)\right)
$$

Так как любые две точки геодезически полного многообразия можно соединить геодезической, для любых двух точек $x, y \in M^{n}$ мы имеем

$$
\lambda_{i}(x) \leqslant \lambda_{i+1}(y)
$$


и первая часть теоремы 1 доказана.

Пусть теперь для каждой точки $y$ некоторого подмножества $V \subset M^{n}$ вьполняется равенство $\lambda_{i}(y)=\lambda_{i+1}(y)$ (для некоторого фиксированного $i \in\{1,2, \ldots, n-1\}$ ). Из первой части теоремы 1 вытекает, что тогда значение $\lambda_{i}$ постоянно для всех точек множества $V$. Обозначим его через $\lambda$. Пусть $\lambda_{i}(x)<\lambda_{i+1}(x)$. Рассмотрим множество $U \subset T_{x} M^{n}$ всех векторов скорости в точке $x$ всех геодезических, соединяющих точку $x$ с хотя бы одной точкой из $V$. По лемме 2 множество $U$ имеет меру нуль. Так как $V$ лежит в образе экспонентциального отображения $\exp _{x}: T_{x} M^{n} \rightarrow M^{n}$ множества $U$, то множество $V$ тоже имеет меру нуль. Теорема 1 доказана.

ДОКАЗАТЕЛЬСТВО ТЕОРЕМЫ 2. Пусть метрики $g, \bar{g}$ геодезически эквивалентны на связном геодезически полном по отношению к метрике $g$ многообразии $M^{n}$. Предположим, что для некоторых точек $x, y \in M^{n}$ и для некоторого числа $i \in\{1,2, \ldots, n-1\}$

$$
\lambda_{i}(x)=\lambda_{i+1}(y)=\lambda .
$$

Если для некоторой точки $z \in M^{n}$ вьполнено $\lambda_{i-1}(z)=\lambda$ (или $\lambda_{i+2}(z)=\lambda$ ), то по теореме 1 для каждой точки $z \in M^{n}$ имеем $\lambda_{i}(z)=\lambda\left(\right.$ соответственно $\left.\lambda_{i+1}(z)=\lambda\right)$ и утверждение теоремы, очевидно, выполнено.

Рассмотрим функцию $F_{\lambda}: T^{*} M^{n} \rightarrow \mathbb{R}$. Пусть для некоторой точки $(z, \nu) \in T^{*} M^{n}$, $\nu \neq 0$, дифференциал $d F_{\lambda}$ равен 0 . Покажем, что тогда хотя бы одно из чисел $\lambda_{i}(z)$, $\lambda_{i+1}(z)$ равно $\lambda$.

Действительно, заменой координат в окрестности точки $z$ приведем метрику $g$ в точке $z$ к единично-диагональному виду, а метрику $\bar{g}$ к диагональному $\operatorname{diag}\left(\rho_{1}(z), \ldots\right.$, $\rho_{n}(z)$ ). Тогда функция $F_{\lambda}$ (как функция на кокасательном пространстве $T_{z}^{*} M^{n}$ ) задается формулой

$$
F_{\lambda}=-\sum_{i=1}^{n} \Pi_{i}(\lambda) p_{i}^{2}
$$

Следовательно, частные производные этой функции по переменным $p_{i}$

$$
\frac{\partial F_{\lambda}}{\partial p_{i}}=-2 \Pi_{i}(\lambda) p_{i}
$$

Поэтому $\lambda$ является одним из собственных значений отображения $B$. По теореме 1 либо $\lambda_{i}(z)=\lambda$, либо $\lambda_{i+1}(z)=\lambda$.

Так как многообразие $M^{n}$ геодезически полно, существует такая геодезическая $\gamma$ : $\mathbb{R} \rightarrow M^{n}$, что $\gamma(0)=x$ и $\gamma(1)=y$. Рассмотрим соответствующую геодезическую траекторию $(\gamma, \dot{\gamma}): \mathbb{R} \rightarrow T M^{n} \cong T^{*} M^{n}$. Покажем, что дифференциал $d F_{\lambda}$ обращается в нуль в каждой точке этой геодезической траектории. Так как функция $F_{\lambda}$ является интегралом геодезического потока метрики $g$, достаточно показать, что дифференциал $d F_{\lambda}$ обращается в нуль в начальной точке $(x, \dot{\gamma}(0))$ этой геодезической траектории. По лемме 1

$$
\lambda=\lambda_{i}(x) \leqslant t_{i}(x, \dot{\gamma}(0)) \quad \text { и } \quad t_{i}(y, \dot{\gamma}(1)) \leqslant \lambda_{i+1}(y)=\lambda .
$$

Так как коэффициенты $I_{k}$ сохраняются вдоль геодезических траекторий,

$$
t_{i}(x, \dot{\gamma}(0))=t_{i}(y, \dot{\gamma}(1))
$$


откуда $t_{i}(x, \dot{\gamma}(0))=t_{i}(y, \dot{\gamma}(1))=\lambda$. Следовательно, геодезическая траектория $(\gamma, \dot{\gamma})$ лежит в топологическом пространстве

$$
Q=\left\{(z, \xi) \in T^{*} M^{n}: F_{\lambda}(z, \xi)=0\right\}
$$

Покажем, что дифференциал $d F_{\lambda}$ равен 0 в точке $(x, \dot{\gamma}(0)) \in Q \cap T_{x}^{*} M^{n}$. Фактически, мы покажем, что никакая окрестность этой точки в топологическом пространстве $Q$ не гомеоморфна $(2 n-1)$-мерному диску. Действительно, по предположению, в каждой точке $z$ некоторой окрестности $U \subset M^{n}$ точки $x$ собственное подпространство ограничения отображения $B$ на $T_{z} M^{n}$ одномерно. Следовательно, для некоторого векторного поля $\phi$ на $U$

$$
B \phi=\lambda_{i} \phi \quad \text { и } \quad g(\phi, \phi)=1 .
$$

Канонически отождествим с помощюю метрики $g$ касательное и кокасательное расслоения к многообразию $M^{n}$. Если мы приведем заменой координат в окрестности точки $z$ метрику $g$ в точке $z$ к единично-диагональному виду, а метрику $\bar{g}$ к диагональному $\operatorname{diag}\left(\rho_{1}(z), \ldots, \rho_{n}(z)\right)$, то $i$-я компонента вектора $\phi$ будет равна 1 или -1 , а все остальные компоненты будут равны 0 . Более того, $i$-я компонента произвольного вектора $\nu \in T_{z} M^{n} \cong T_{z}^{*} M^{n}$ равна с точностью до знака скалярному произведению $g(\nu, \phi)$.

Для будущего заметим, что коэффициенты многочлена $\Pi_{i}(t)$ из формулы $(6)$ выражаются через характеристические инварианты отображения $B$ и через $\lambda_{i}$ и, следовательно, гладко зависят от точки окрестности $U$. Для того, чтобы подчеркнуть зависимость многочлена $\Pi_{i}$ от точки $z \in U$, мы будем писать $\Pi_{i}(t ; z)$ вместо $\Pi_{i}(t)$.

Рассмотрим функцию $I: T M^{n} \rightarrow \mathbb{R}, I(z, \nu)=g(\nu, \phi)$. Очевидно, $I(x, \dot{\gamma}(0))=0$ и частная производная $\partial I / \partial p_{i}$ отлична от нуля в точке $(x, \dot{\gamma}(0))$. По теореме о неявной функции некоторая окрестность $V$ точки $(x, \dot{\gamma}(0))$ в топологическом пространстве

$$
Q^{*}=\left\{(z, \nu) \in T M^{n}: I(z, \nu)=0\right\}
$$

гомеоморфна прямому произведению $U^{\prime} \times D^{n-1}$, где $U^{\prime} \subset U$ является окрестностью точки $x$, а $D^{n-1}$ является $(n-1)$-мерным диском.

При этом ограничение проекции $\pi$ на $V$ совпадает с естественной проекцией прямого произведения $U^{\prime} \times D^{n-1}$ на компоненту $U^{\prime}$.

Для каждой точки $(z, \nu) \in V$ рассмотрим точки

$$
\left(z, \nu_{+}\right)=\left(z, \nu+\phi \sqrt{\frac{F_{\lambda}(z, \nu)}{\Pi_{i}(\lambda ; z)}}\right), \quad\left(z, \nu_{-}\right)=\left(z, \nu-\phi \sqrt{\frac{F_{\lambda}(z, \nu)}{\Pi_{i}(\lambda ; z)}}\right) .
$$

Так как $\lambda_{i}(z) \leqslant \lambda<\lambda_{i+1}(z)$, подкоренное выражение всегда неотрицательно. Оно равно 0 , только если $\lambda_{i}(z)=\lambda$. Легко увидеть, что если для точек $\left(z^{1}, \nu^{1}\right),\left(z^{2}, \nu^{2}\right) \in V$ выполняется хотя бы одно из равенств

$$
\begin{array}{ll}
\left(z^{1}, \nu_{+}^{1}\right)=\left(z^{2}, \nu_{+}^{2}\right), & \left(z^{1}, \nu_{-}^{1}\right)=\left(z^{2}, \nu_{+}^{2}\right) \\
\left(z^{1}, \nu_{+}^{1}\right)=\left(z^{2}, \nu_{-}^{2}\right), & \left(z^{1}, \nu_{-}^{1}\right)=\left(z^{2}, \nu_{-}^{2}\right)
\end{array}
$$

то $\left(z^{1}, \nu^{1}\right)=\left(z^{2}, \nu^{2}\right)$. 
Непосредственно проверяется, что $F_{\lambda}\left(z, \nu_{+}\right)=F_{\lambda}\left(z, \nu_{-}\right)=0$. С другой стороны, любая точка $(z, \xi)$ некоторой окрестности точки $(x, \dot{\gamma}(0))$ в топологическом пространстве $Q$ является либо точкой $\left(z, \nu_{+}\right)$, либо точкой $\left(z, \nu_{-}\right)$для точки $(z, \nu)=(z, \xi-\phi g(\phi, \xi))$ $\in V$. Следовательно, некоторая окрестность точки $(x, \dot{\gamma}(0))$ в топологическом пространстве $Q$ гомеоморфна прямому произведению двух экземпляров $U^{\prime}$, склеенных по точкам $z$, в которых $\lambda_{i}(z)=\lambda$, на диск $D^{n-1}$. Так как $\lambda_{i}(x)=\lambda$, никакая окрестность точки $(x, \dot{\gamma}(0))$ в топологическом пространстве $Q$ не гомеоморфна диску. Следовательно, дифференциал $d F_{\lambda}$ равен 0 в точке $(x, \dot{\gamma}(0))$. Следовательно, он равен 0 в каждой точке геодезической $(\gamma, \dot{\gamma})$. Таким образом, каждая точка вещественной прямой $\mathbb{R}$ лежит в одном из следующих подмножеств:

$$
\begin{aligned}
& \Gamma_{0}=\left\{\tau \in \mathbb{R}: \lambda_{i}(\gamma(\tau))=\lambda\right\} \\
& \Gamma_{1}=\left\{\tau \in \mathbb{R}: \lambda_{i+1}(\gamma(\tau))=\lambda\right\}
\end{aligned}
$$

Эти подмножества очевидно замкнуты и непусты. Следовательно, их пересечение не является пустым множеством. По определению, в любой точке $\tau$ этого пересечения $\lambda_{i}(\gamma(\tau))=\lambda_{i+1}(\gamma(\tau))=\lambda$. Теорема доказана.

ДоКАЗАТЕЛЬСТВо ТЕОРЕМЫ 3. Пусть многообразие $M^{n}$ связно и замкнуто, пусть метрики $g, \bar{g}$ на многообразии $M^{n}$ геодезически эквивалентны и пусть собственные значения $\rho_{1}(x), \rho_{2}(x), \ldots, \rho_{n}(x)$ различны в каждой точке многообразия. Тогда по теореме 2 существуют такие числа $\tau_{1}<\tau_{2}<\cdots<\tau_{n-1}$, что для любой точки $x \in M^{n}$

$$
\lambda_{1}(x)<\tau_{1}<\lambda_{2}(x)<\tau_{2}<\cdots<\tau_{n-1}<\lambda_{n}(x) .
$$

Рассмотрим многочлен

$$
-\left(t-\tau_{1}\right)\left(t-\tau_{2}\right) \cdots\left(t-\tau_{n-1}\right)=C_{n-1} t^{n-1}+C_{n-2} t^{n-2}+\cdots+C_{0} .
$$

Рассмотрим подмножество

$$
L^{n}=\left\{(x, \xi) \in T^{*} M^{n}: I_{0}(x, \xi)=C_{0}, I_{1}(x, \xi)=C_{1}, \ldots, I_{n-1}(x, \xi)=C_{n-1}\right\} .
$$

Мы покажем, что в каждой точке этого подмножества дифференциалы

$$
d I_{0}, d I_{1}, \ldots, d I_{n-1}
$$

линейно независимы, так что по теореме Лиувилля (см., например, [14]) это подмножество состоит из набора торов Лиувилля, и что ограничение проекции $\pi: T^{*} M^{n} \rightarrow M^{n}$, $\pi(x, \xi)=x$, на любой из этих торов Лиувилля не имеет особых точек и, тем самым, является накрытием многообразия $M^{n}$.

Достаточно показать, что в каждой точке $(x, \xi) \in L^{n}$ определитель $(n \times n)$-матрицы

$$
W_{i j}=\frac{\partial I_{i}}{\partial p_{j}}
$$

отличен от нуля. Действительно, в этом случае дифференциалы (10) линейно независимы. По теореме о неявной функции ограничение проекции $\pi$ на $L^{n}$ регулярно в точке $(x, \xi)$. Обозначим через $\lambda_{1}<\lambda_{2}<\cdots<\lambda_{n}$ собственные значения отображения 
Синюкова в точке $x$. Так как функции $F_{\lambda_{1}}, F_{\lambda_{2}}, \ldots, F_{\lambda_{n}}$ являются линейными комбинациями функций $I_{0}, I_{1}, \ldots, I_{n-1}$, достаточно показать, что определитель $(n \times n)$-матрицы

$$
\widetilde{W}_{i j}=\frac{\partial F_{\lambda_{i}}}{\partial p_{j}}
$$

не обращается в нуль в точках множества $L_{n}$.

Заменой координат в окрестности точки $x$ приведем метрику $g$ в точке $x$ к единично-диагональному, а метрику $\bar{g}$ к диагональному виду. Тогда функция $F_{t}$ задана формулой (6) и, следовательно, матрица $\widetilde{W}$ является диагональной матрицей

$$
\operatorname{diag}\left(-2 \Pi_{1}\left(\lambda_{1}\right) p_{1},-2 \Pi_{2}\left(\lambda_{2}\right) p_{2}, \ldots,-2 \Pi_{n}\left(\lambda_{n}\right) p_{n}\right) .
$$

Так как по условию

$$
\lambda_{1}(x)<\tau_{1}<\lambda_{2}(x)<\tau_{2}<\cdots<\tau_{n-1}<\lambda_{n}(x),
$$

все числа вида $\Pi_{i}\left(\lambda_{i}\right) p_{i}$ отличны от нуля; отсюда следует, что определитель матрицы (11) отличен от нуля. Теорема доказана.

\section{СПИСОК ЦИТИРОВАННОЙ ЛИТЕРАТУРЫ}

[1] Микеш Й. Геодезические отображения аффинно-связных и римановых пространств // Итоги науки и техники. Современная матем. и ее прилож. Т. 11. Геометрия-2. М.: ВИНИТИ, 1994.

[2] Синюков Н. С. К теории геодезических отображений // Докл. АН СССР. 1966. Т. 169. C. $770-772$.

[3] Matveev V.S., Topalov P. J. Quantum integrability of the Beltrami-Laplace operator as geodesic equivalence // Math. Z. 2001. V. 238. № 4. P. 833-866.

[4] Матвеев В. С., Топалов П. Й. Геодезическая эквивалентность метрик на поверхностях как интегрируемость их геодезических потоков // Докл. РАН. 1999. Т. 367. № 6. С. 736-738.

[5] Болсинов А. В., Фоменко А. Т. Римановы метрики с интегрируемыми геодезическими потоками на поверхностях: локальная и глобальная геометрия // Матем. сб. 1998. Т. 189. № 10. C. $5-32$.

[6] Igarashi M., Kiyohara K., Sugahara K. Noncompact Liouville surfaces // J. Math. Soc. Japan. 1993. V. 45. № 3. P. 460-479.

[7] Matveev V.S. Geschlossene hyperbolische 3-Mannigfaltigkeiten sind geodätsich starr // Manuscripta Math. 2001. V. 105. № 3. P. 343-352.

[8] Matveev V.S. Three-manifolds admitting metrics with the same geodesics // Math. Res. Lett. 2002. V. 9. № 2-3. P. 267-276.

[9] Matveev V.S. Low-dimensional manifolds admitting metrics with the same geodesics // Contemporary Mathematics. 2002. V. 308. P. 229-243.

[10] Matveev V.S., Topalov P. J. Trajectory equivalence and corresponding integrals // Regular and Chaotic Dynamics. 1998. V. 3. P. 29-44.

[11] Matveev V.S., Topalov P. J. Geodesic equivalence via integrability // Geometriae Dedicata. 2003. V. 96. P. 91-115.

[12] Bolsinov A. V., Fomenko A.T., Matveev V.S. Riemannian metrics with integrable geodesic flows on surfaces: local and global geometry // Max-Planck-Institute for Mathematics Preprint Series № 120. Bonn: MPIM, 1998.

[13] Topalov P.J., Matveev V.S. Geodesic equivalence and integrability // Max-PlanckInstitute for Mathematics Preprint Series № 74. Bonn: MPIM, 1998.

[14] Арнольд В. И. Математические методы классической механики. М.: Наука, 1979.

Челябинский государственный университет 